\title{
Assessing risks of genetic engineering
}

Sir-Miller et al. are right to draw attention to difficulties that might be encountered in considering socioeconomic issues before the release of genetically modified organisms (Nature $392,221 ; 1998)$. In fact, it is possible to foresee many more. It may also be the case that "such efforts would constitute major interdisciplinary research projects and would be highly vulnerable to the vagaries of value judgements", although these are problems that do not preclude productive work in other areas of policy research. (One of Miller et al.'s examples, the automobile, is increasingly attracting interdisciplinary scrutiny of precisely the type they eschew.)

However, arguments for the complexity of these issues per se can hardly be taken to detract from the importance of efforts that should be made to address them head on. Social and economic factors surely merit overt consideration. We argue, simply, that it is less desirable still to leave them vulnerable to the vagaries of value judgements made tacitly under the constraints of risk assessment frameworks that fail to take account of the need for interdisciplinary input (see Nature 391, 528; 1998).

Miller et al. claim that consideration of socioeconomic issues would necessarily entail that "any field trial, anywhere... would be subject to an evaluation of possible social and economic impact anywhere else in the world". With regard to the 'biosafety protocol', one option under consideration (article 26) would require the assessment of socioeconomic impact before the transboundary movement of a genetically modified organism. This would not entail that India, for example, need consider the potential economic impact upon Texan farmers of a new variety of genetically modified sorghum, before commencing field trials in Andhra Pradesh. (Whether or not the impact upon farmers in India is considered would of course depend upon Indian legislation or guidelines.)

Finally, Miller et al. allude to the stipulations of free trade agreements. The interaction of the 'biosafety protocol' with such agreements is moot. However, there are important precedents. The Montreal protocol (to control the release of ozonedepleting chemicals) is both appropriate and useful, despite its possible conflict with free trade.

\section{Tom Crompton}

UNIDO/ICGEB Liaison Office,

Vienna International Centre,

Austria

e-mail:tcrompton@unido.org

John Barrett

Department of Genetics,

University of Cambridge, UK

Chris Gliddon

University of Wales, Bangor, UK

Julian Kinderlerer

Sheffield Institute of Biotechnological

Law and Ethics, University of Sheffield, UK

George Tzotzos

UNIDO Investment and Technology

Promotion Division,

Technology Service, Vienna, Austria

Sir - There seems to be a willingness among many scientists involved in genetic manipulation to speculate favourably at an early stage on the potential economic value of discoveries, with far less enthusiasm for considering potential risks.

Consider this. How often have you seen a grant application or paper in this field that makes a favourable value judgement on the potential value of the work in the concluding paragraph? And how often have you seen similar documents that contain statements giving equal weight to potential biosafety issues or possible adverse economic outcomes? The letter by Miller et al. seems to be another manifestation of this mindset, conveying the underlying assumption that there is no justification for attempting to predict wider negative impacts of genetically manipulated organisms that appear superficially to address pressing environmental, health or food needs.

Miller et al.'s scepticism about the value of interdisciplinary research projects aimed at investigating socioeconomic impacts of recombinant organisms during the early phases of field testing overlooks at least one potentially valuable outcome of this work. Such research, predicting the potential impact of changes at the earliest opportunity, would maximize the time available for affected communities to develop contingency measures to buffer the socioeconomic impact of change. Sudden, unpredicted economic changes triggered by the largescale introduction of genetically manipulated organisms, with no thought for contingencies to mitigate undesirable effects, may be politically and socially destabilizing, particularly in developing countries.

Their argument for dismissing the need for such research - that similar analyses were not required for the introduction of earlier novel technologies such as the internal combustion engine - appears to be breathtakingly complacent and the antithesis of the spirit of scientific enquiry and advancement. Why not extend such logic to safety regulations for all new agrochemicals, and campaign for the retention of standards applied in the nineteenth century? It is perfectly understandable that the genetic engineering community should present an upbeat image of its work to reassure investors, but it is also in their interests to take a longer view of the impact of genetically manipulated organisms. This will help to reassure the public that science can take an active stance on a wide spectrum of biosafety issues.

\section{Phil Gates}

Department of Biological Sciences,

Universty of Durham,

Durham DH1 3LE, UK

e-mail:p.j.gates@durham.ac.uk

\section{Who flew first?}

Sir - J. L. Heilbron and W. F. Bynum (Nature 391, 13-16; 1998), in "Eighteenninety-eight and all that", celebrate some interesting anniversaries. The choice of such anniversaries is always subjective, because there are so many, but I should like to mention some birth dates that I would have included: 1548 Simon Stevin (Flemish) and Giordano Bruno (Italian); 1598 Bonaventura Cavalieri (Italian); 1698
Thomas Hodgkin (English) and the Frenchmen Charles du Fay, Pierre Bouguer and P. L. Moreau de Maupertuis; 1748 Jean Bernoulli (Swiss) and C. L. Berthollet (French); 1798 Auguste Comte (French).

J. Berzelius (Swedish), B. Bolzano (Czechoslovak) and Caroline Herschel (German) died in 1848, and the Frenchman Marin Mersenne in 1648.

Maybe I can use the authors' bias as an excuse for my own nationalistic and last comment: how can one mention the Wright brothers as the inventors of the aeroplane without referring to the controversies on this subject? For example, why not a simple allusion to the Brazilian aviation pioneer Alberto Santos Dumont or to the

Frenchman Clément Ader?

Ildeu de Castro Moreira

Instituto de Física - UFRJ,

CP 68528, Ilha do Fundão,

21945-170 Rio de Janeiro, Brazil

e-mail: ildeu@if.ufrj.br 